\title{
When the situation almost cannot be worse...
}

\author{
Petr Hájek, Radka Adlová, Josef Veselka \\ Department of Cardiology, University Hospital Motol, Prague, Czech Republic
}

Adv Interv Cardiol 2018; 14, 3 (53): 219-220

DOI: https://doi.org/10.5114/aic.2018.78321

A 60-year-old man without a history of coronary artery disease presented with ST-elevation myocardial infarction (STEMI). During transportation to the hospital, he developed ventricular fibrillation and later pulseless electrical activity. Chest compressions with an automated mechanical compression-decompression device (aMCD) were initiated. Coronary angiography showed total occlusion of the unprotected left main coronary artery (ULM), and primary percutaneous coronary intervention $(\mathrm{PCl})$ was performed during continuous cardiopulmonary resuscitation (CPR). After the reperfusion, the patient's heart started to generate effective contractions and aMCD could be discontinued. Return of spontaneous circulation was achieved after 90 min of cardiac arrest. The patient died of cardiogenic shock $11 \mathrm{~h}$ later [1].

This is one of several potential clinical scenarios of patients with uLM occlusion. Reperfusion was performed in a timely manner, but the patient still died. What are the causes of such unfavorable progress? Is there any possibility of discovering how to increase the chance of surviving this critical situation?

The authors of the article "Acute, total occlusion of the left main stem: coronary intervention options, outcomes, and recommendations" published in the current issue of "Advances in Interventional Cardiology/Postępy w Kardiologii Interwencyjnej" presented their own experience with this uncommon but often catastrophic event. In a group of 23 patients, they found that these patients frequently experience cardiogenic shock (87\%), after or during CPR (52\%), and even with a procedural success rate of $91 \%$, the in-hospital and 6-month mortality rates were high ( $57 \%$ and $65 \%$, respectively).

Patients with ULM lesions represent a subgroup of acute coronary syndrome patients with the largest amount of jeopardized myocardium. They therefore have higher mortality and a higher probability of developing cardiogenic shock. In a meta-analysis of a group of 977 patients with $\mathrm{PCl}$ for acute myocardial infarction (AMI) due to uLM disease, $26 \%$ of patients were in cardiogenic shock. The 30 -day mortality rate was $55 \%$ and $11 \%$, respectively, for those with or without cardiogenic shock [2].

Severe hemodynamic instability of patients with uLM occlusion can result in the necessity for CPR before or during $\mathrm{PCl}$. Type and length of CPR influences performance of the revascularization procedure. A mechanical chest compression device has some advantages over manual chest compression. It is easy to use and independent of physical activity and fatigue of rescuers. Most importantly, it enables uninterrupted chest compressions during $\mathrm{PCI}$. In the presented study, aMCDs were used in 7 of 12 resuscitated patients. The results of the Štěchovskỳ et al. study suggest that use of aMCD can be associated with a risk of myocardial contusion compared with manual compressions [3]. Although a causal relationship between contusion and outcomes has not been proven, this possibility should be kept in mind during care after CPR with aMCD.

Percutaneous revascularization of an occluded $\mathrm{ULM}$ provides revascularization of threatened myocardium less invasively and first of all more rapidly compared with coronary artery bypass graft (CABG) surgery. Especially in patients in cardiogenic shock or even in resuscitated patients, there is no time to consider options other than immediate percutaneous revascularization. No randomized trial has been conducted comparing PCI with CABG in the setting of AMI due to occlusion of the uLM. It is unlikely that such a study will ever be performed in the future. Data from non-randomized studies and registries are biased because patients in cardiogenic shock are more likely to undergo $\mathrm{PCl}$ rather than $\mathrm{CABG}$ due to their preoperative instability [4].

Aspiration thrombectomy can be an effective initial strategy for rapid reperfusion and thrombus burden decrease [5], although routine thrombus aspiration in STEMI did not improve long-term clinical outcomes and might be associated with an increased risk of stroke [6].

\section{Corresponding author:}

Petr Hájek MD, Department of Cardiology, University Hospital Motol, V Uvalu 84, 15000 Praha 5, Czech Republic, phone: +42 0603280604, e-mail: petr.hajek@fnmotol.cz

Received: 30.08.2018, accepted: 30.08.2018. 
Several percutaneous techniques of ULM intervention are available, with promising results in randomized trials, but the acute situation of ULM occlusion requires a rapid and simple solution that probably offers provisional stenting. Complex bifurcation strategies require more balloon inflations and increased consumption of contrast dye, which can contribute to further hemodynamic deterioration. On the other hand, final intravascular ultrasound evaluation of results can be a useful tool for its optimization. There was a mean stent diameter of $3.3 \mathrm{~mm}$ in the reported study, and from other studies we know that in $75 \%$ of cases, the $L M$ is $>4.0 \mathrm{~mm}$ in diameter and averages $4.75 \mathrm{~mm}$ [7]. Diffuse vasospasm, frequently present in patients requiring catecholamine support, can cause LM diameter underestimation, and a suboptimally dilated stent can contribute to the risk of subacute stent thrombosis.

Performance of $\mathrm{PCl}$ is mandatory for reperfusion to salvage ischemic myocardium. Data on acute LM intervention (including the present study) indicated a procedural success of more than 90\% [4]. Reperfusion is also associated with an additional injury that is partially reversible (stunning), but also irreversible and leads to increased infarct size and microvascular dysfunction. Several methods of ischemic conditioning have been identified as complex processes underlying the cardioprotection in experimental studies. Attempts to translate conditioning strategies and drug therapy into the clinical setting mostly have failed to reduce infarct size and improve clinical outcomes in STEMI patients [8] so far. The large amount of myocardium at risk in patients with uLM occlusion represents a large territory for ischemia/ reperfusion injury, and clearly such patients would most benefit from cardioprotection.

Percutaneous mechanical circulatory support (MCS) devices, such as the intra-aortic balloon pump (IABP), Impella (Abiomed Inc., Danvers, Massachusetts), and extracorporeal membrane oxygenation (ECMO), may help to overcome the critical phase after successful PCl, when ischemia/reperfusion injury persists. These devices can also help to decrease the dose of vasopressors and inotropes and thus reduce the possible risk of their cardiotoxicity. The intra-aortic balloon pump, although most frequently used, provides minimal hemodynamic assistance that is insufficient to support the circulation in severe forms of cardiogenic shock. It may be reasonable to use an IABP while performing $\mathrm{PCl}$ in preshock situations. Device escalation is required if the initial support device does not improve hemodynamics. For patients with shock, MCS with the Impella is recommended. For patients with profound cardiopulmonary failure including respiratory failure or ongoing cardiopulmonary resuscitation, the use of ECMO should be considered [9]. Although improvement in hemodynamic parameters with the use of current MCS has been observed, a mortality benefit has not been proven yet in multicenter randomized trials. Therefore, the use of MCS should be limited to carefully selected (the sickest) patients.

Occlusion of the LM is the most serious coronary finding, with a high risk of cardiogenic shock development and related mortality. Although a successful $\mathrm{PCl}$ is an indispensable condition, a multidisciplinary heart team including critical care physicians, anesthesiologists, and cardiothoracic surgeons plays an essential role in postprocedure management. Therapeutic success depends on correct timing of particular steps, advanced skills of operators, availability of medical devices including MCS and quality of intensive care. Nevertheless, the prognosis of patients with ULM occlusion remains uncertain, with high mortality even with optimal management. But when the situation almost cannot be worse, we are challenged to use any available option to save a patient's life.

\section{Conflict of interest}

The authors declare no conflict of interest.

\section{References}

1. Stechovsky C, Hajek P, Cipro S, Veselka J. Mechanical chest compressions in prolonged cardiac arrest due to ST elevation myocardial infarction can cause myocardial contusion. Int J Angiol 2016; 25: 186-8.

2. Vis MM, Beijk MA, Grundeken MJ, et al. A systematic review and meta-analysis on primary percutaneous coronary intervention of an unprotected left main coronary artery culprit lesion in the setting of acute myocardial infarction. JACC Cardiovasc Interv 2013; 6: 317-324.

3. Štěchovský C, Hájek P, Cipro Š, Veselka J. Risk of myocardial contusion in cardiac arrest patients resuscitated with mechanical chest compression device. Int J Cardiol 2015; 182: 50-1.

4. Lee MS, Dahodwala MQ. Percutaneous coronary intervention for acute myocardial infarction due to unprotected left main coronary artery occlusion: status update 2014. Catheter Cardiovasc Interv 2015; 85: 416-20.

5. Hajek P, Alan D, Vejvoda J, et al. Treatment of a large left main coronary artery thrombus by aspiration thrombectomy. J Thromb Thrombolysis 2009; 27: 352-4.

6. Jolly SS, Cairns JA, Yusuf S, et al. Outcomes after thrombus aspiration for ST elevation myocardial infarction: 1-year follow-up of the prospective randomised TOTAL trial. Lancet 2016; 387: 127-35.

7. Rab T, Sheiban I, Louvard Y, et al. Current interventions for the left main bifurcation. JACC Cardiovasc Interv 2017; 10: 849-65.

8. Heusch G, Gersh BJ. The pathophysiology of acute myocardial infarction and strategies of protection beyond reperfusion: a continual challenge. Eur Heart J 2017; 38: 774-84.

9. Atkinson TM, Ohman EM, O'Neill WW, et al. Interventional Scientific Council of the American College of Cardiology. A practical approach to mechanical circulatory support in patients undergoing percutaneous coronary intervention: an interventional perspective. JACC Cardiovasc Interv 2016; 9: 871-83. 